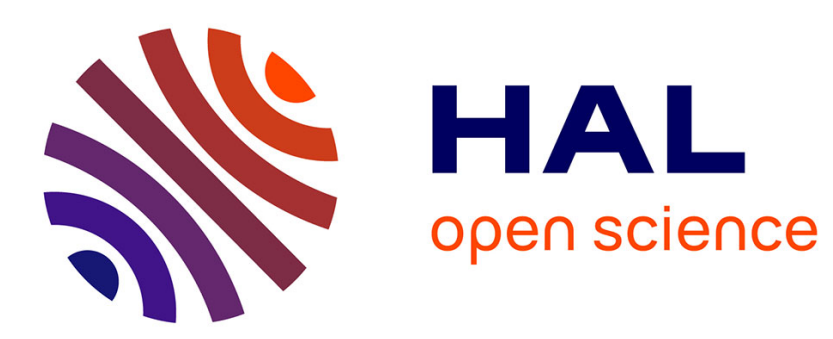

\title{
User Association and Resource Allocation Optimization in LTE Cellular Networks
}

Nessrine Trabelsi, Chung Shue Chen, Rachid El-Azouzi, Laurent Roullet, Eitan Altman

\section{- To cite this version:}

Nessrine Trabelsi, Chung Shue Chen, Rachid El-Azouzi, Laurent Roullet, Eitan Altman. User Association and Resource Allocation Optimization in LTE Cellular Networks. IEEE Transactions on Network and Service Management, 2017, 14 (2), pp.429 - 440. 10.1109/TNSM.2017.2677778 • hal-01536128

\section{HAL Id: hal-01536128 \\ https://hal.inria.fr/hal-01536128}

Submitted on 10 Jun 2017

HAL is a multi-disciplinary open access archive for the deposit and dissemination of scientific research documents, whether they are published or not. The documents may come from teaching and research institutions in France or abroad, or from public or private research centers.
L'archive ouverte pluridisciplinaire HAL, est destinée au dépôt et à la diffusion de documents scientifiques de niveau recherche, publiés ou non, émanant des établissements d'enseignement et de recherche français ou étrangers, des laboratoires publics ou privés. 


\title{
User Association and Resource Allocation Optimization in LTE Cellular Networks
}

\author{
Nessrine Trabelsi ${ }^{1,2}$, Chung Shue Chen $^{2}$, Rachid El Azouzi ${ }^{3}$, Laurent Roullet ${ }^{2}$, and Eitan Altman ${ }^{1,4,5}$ \\ ${ }^{1}$ INRIA, 06902 Sophia Antipolis Cedex, France \\ ${ }^{2}$ Bell-Labs, Nokia, Centre de Villarceaux, 91620 Nozay, France \\ ${ }^{3}$ LIA-CERI, University of Avignon, 339 chemin des Meinajaries, Avignon cedex 9, France \\ ${ }^{4}$ UCA - University of Cte d'Azur \\ ${ }^{5}$ LINCS, 23 Ave d'Italie, Paris 75013, France \\ Email: \{nessrine.trabelsi, eitan.altman\}@inria.fr, \{chung_shue.chen, laurent.roullet\}@ nokia.com, \\ rachid.elazouzi@univ-avignon.fr
}

\begin{abstract}
As the demand for higher data rates is growing exponentially, homogeneous cellular networks have been facing limitations when handling data traffic. These limitations are related to the available spectrum and the capacity of the network. Heterogeneous Networks (HetNets), composed of Macro Cells (MCs) and Small Cells (SCs), are seen as the key solution to improve spectral efficiency per unit area and to eliminate coverage holes. Due to the large imbalance in transmit power between MCs and SCs in HetNets, intelligent User Association (UA) is required to perform load balancing and to favor some $\mathrm{SCs}$ attraction against MCs. As Long Term Evolution (LTE) cellular networks use the same frequency sub-bands, User Equipments (UEs) may experience strong Inter-Cell Interference (ICI), especially at cell edge. Therefore, there is a need to coordinate the Resource Allocation (RA) among the cells and to minimize the ICI. In this paper, we propose a generic algorithm to optimize user Association and resource allocation in LTE networks. Our solution, based on game theory, permits to compute Cell Individual Offset (CIO) and a pattern of power transmission over frequency and time domain for each cell. Simulation results show significant benefits in the average throughput and also cell edge user throughput of $40 \%$ and $55 \%$ gains respectively. Furthermore, we also obtain a meaningful improvement in energy efficiency.
\end{abstract}

Index Terms-inter-cell interference coordination, user association, resource allocation, cell individual offset, almost blank sub-frames, power control, frequency sub-band, optimization.

\section{INTRODUCTION}

Till the past few years, homogeneous LTE cellular networks, composed of identical Base Stations (BS) called macro BSs, managed to optimize the coverage and to handle the data traffic generated by the users. Generally, the deployment of these macro BSs is planned in a way that minimizes the overlap between the cells and at the same time guarantees a continuous coverage for all users in the network. However, because of the exponential increase in the number of connected devices, the rapid growth of data traffic and the demand for higher data rates, LTE networks have been facing great difficulty to handle the data amount, especially in the most crowded environments and at cell edges. These limitations are related to the available spectrum and network capacity bound. The first element to increase channel capacity is bandwidth. As spectrum is scarce, the acquisition of licensed bands is often very expensive, at least for the time being. Network operators prefer to use the available licensed spectrum more efficiently. Another approach consists of enhancing the macro network layer efficiency through some technology upgrades. For instance, the performance of these networks can be improved thanks to advancement in the air interface, using multi-antenna techniques and implementing more efficient modulation and coding schemes.

Cell size is another factor that affects the number of users that we can support by the base stations. One of the most wellknown capacity-enhancing strategies is the use of smaller cells. This permits to increase the frequency reuse, also known as cell-splitting gain. The macrocell network can also be densified by adding more sectors per macro site or by deploying more BSs. However, it becomes more difficult and expensive to find new macro sites. Based on cell densification, HetNets has been proposed by the 3rd Generation Partnership Projects (3GPPs) to cope with the limited amount of spectrum. What is HetNets and how does it improve the network capacity?

The idea behind HetNets is to overlay existing homogeneous LTE networks, commonly called the macro layer, with additional smaller power low-complexity base stations using the same spectrum to increase the bit rate per unit area. Small cells (e.g., femto, pico, micro, metro cells, etc.) have coverage range that varies from a few meters to several hundreds of meters, in contrast to the tens of kilometers covered by macro cells. Since coverage is already provided by the macro BSs, small cells are often placed in densely populated areas to boost the capacity of LTE network.

In Orthogonal Frequency Division Multiple Access (OFDMA) cellular network, the physical radio resources are partitioned into a time-frequency grid. In HetNets, small cells, placed on top of the macro layer, use the same time-frequency resources as the macro cells. As a result, the reuse of resources in frequency, time and space is highly increased and thus the spectrum efficiency of the network is improved. The densification of the network results in reducing the average distances 
between a user and the nearest BS and thus decreasing the pathloss experienced by the transmitted signal and improving the link gain and the capacity of the channel. This permits to offload the users from MCs to SCs, to balance the traffic load and also to increase the overall network throughput and efficiency. HetNets has extra benefits as it permits to reduce the complexity and the cost of new deployments. Another advantage of this architecture is the lower transmit power of the small cells which would lead to reducing the carbon footprint of mobile networks.

While considered as the most attractive way to improve the network capacity, introducing a mix of various cell sizes with different transmission powers adds to the complexity of network planning. In traditional cellular networks with frequency reuse equal to 1 (reuse-1), users usually associate with the cell that provides the strongest received signal. The measure of the signal strength depends essentially on the transmission power of the cell and the pathloss encountered. In homogeneous cellular networks where the BSs have similar transmission power, the user association is determined by the pathloss, i.e., mainly by the user-BS distance. In HetNets, with high-power nodes in the macro cells (e.g., 40 Watts) and lowpower nodes in the small cells (e.g., 1 Watt), only few users would attach to these small cells. It may happen that a user, which is closer to a Small Cell (SC) and has a low pathloss compared to that with a Macro Cell (MC), has a stronger signal strength from the $\mathrm{MC}$ as the $\mathrm{MC}$ transmits with a larger power, and thus would attach to the MC, instead of the closer SC. Attaching to the strongest signal in such cases is often sub-optimal or even negative to the system performance since we may under-utilize the small cells. To address this problem, one can systematically expand the area served by the small cell. This mechansim is called Cell Range Extension (CRE) in 3GPP. To offload MCs and associate users with SCs, a Cell Individual Offset is added to the users' Reference Signal Received Power (RSRP) measurements. This would enforce some users, especially those in SC edge, to associate with their nearest SC instead of the MC, inducing a better load balancing in the network. One question that we will address in this paper is the optimization of CIO values and the user association.

Another challenge is to coordinate interference and thus enhance the Signal-to-Interference-plus-Noise Ratio (SINR). Note that in LTE OFDMA system, the whole bandwidth is divided into physical resource blocks in frequency subcarrier and time slot that are orthogonal to each other. Thus, the intra-cell interference is insignificant. However, inter-cell interference is usually severe due to the practice of reuse-1 cellular networks [2]. It could happen that neighboring macro and small cells use the same Resource Blocks (RBs) and result in high inter-cell interference, which is even more severe to cell edge users. Reducing inter-cell interference is necessary. Furthermore, using cell range extension in HetNets could also generate higher inter-cell interference, especially for the users that change their attachment from macro to small cells.

It is vital to ensure that the reuse of the spectrum does not lead to high interference scenarios in LTE networks. To mitigate the inter-cell interference, 3GPP LTE standard has introduced Inter-Cell Interference Coordination (ICIC) and enhanced ICIC (eICIC) methods in Release 8 and Release 10 specifications, respectively. They are provided to address interference issues in HetNets and mitigate interference on data traffic and control channels. Generally, the ICIC techniques are limited to the frequency and/or power domain, for example splitting the frequency bandwidth into parts for adjacent cells or having their transmissions with different power levels. In addition, eICIC focuses on time domain solution through for example Almost Blank Sub-frames (ABS), which is to mute a cell during specific time slots so that its neighboring cells could transmit under minimal interference. It should be also noted that there is an interplay among user association, resource allocation, and interference management. These should be performed in an intelligent manner to improve the user throughput and support high spatial reuse in LTE networks.

In this paper, we will present a framework to optimize LTE networks in user association and dynamic radio resource sharing, and to coordinate the inter-cell interference for enhancing overall network utility. Our work is based on a two-tier model that permits the separation of some control decisions among the eNodeBs (eNBs) and the centralized entity, called the eNB coordinator. The latter receives periodically, via $\mathrm{S} 1$ interface [2], updated measurements from the eNBs. Then, it performs a global optimization to select the best CIOs for user association, to coordinate the allocation of subset of frequency resources to the eNBs, and to adjust the transmission power on each frequency sub-band. This optimization, based on an iterative algorithm and considering the state of the whole network, results in the best CIO, frequency and power settings for each cell. These values are then sent to the eNBs and to be used by their LTE local schedulers for transmissions. Coordinating the transmission power and frequency reuse across cells allows to limit the interference experienced by the mobile users and to improve the quality-of-service (QoS) over the network and also cell edge. It could also yield higher energy efficiency.

The contributions of this paper are listed below:

- We formulate the user association and inter-cell interference problem using a potential game.

- We provide a dynamic solution of user association and inter-cell interference coordination optimizing the Cell Individual Offset (CIO), and transmission power over time and frequency domains to maximize the network utility.

- We provide an analytical investigation of the algorithm and comprehensive performance study. Simulation results have shown significant improvement in the user throughputs and also energy efficiency.

The rest of the paper is organized as follows. Section II presents the related work. In Section III, we define the system model and formulate the user association and interference management problem. We describe the proposed solution and its technical implementation in Section IV. In Section V, we describe the simulation settings and show the numerical results. Finally, Section VI draws the conclusion and highlights some potential future work. 


\section{RELATED WORK}

Inter-cell interference coordination has been the subject of intensive research and standardization and numerous papers have been published on ICIC techniques for LTE OFDMA networks. [5] surveys various ICIC schemes used to mitigate inter-cell interference in OFDMA based cellular networks for enhancing cell edge data rates and the overall network capacity. ICIC techniques are mostly limited to the frequency/power domain where there is a partial use of frequency resources or adaptation of power levels. Previous work has focused on static approaches such as partial and soft frequency reuse [6]. Although these techniques can be easily implemented, they cannot cope with changes in data traffic.

To support increasing data traffic in mobile networks and to address LTE HetNets challenges, 3GPP Release 10 [1] introduced enhanced ICIC techniques based on time resource partitioning to mute macro cells for certain sub-frames and to limit the interference to small cell edge users. In [12], the authors present a comprehensive introduction of eICIC in HetNets and [15] provides a survey on different eICIC techniques and a summary of the evolution of LTE standards.

A comprehensive study of the evolution of interference management techniques from simple ICIC to time domain enhanced inter-cell interference coordination and also advanced coordinated multipoint transmissions (CoMP) is given in [7] and [9]. Note that CoMP was brought by 3GPP LTE-Advanced Release 11, which permits to dynamically coordinate the transmission and reception of signals at multiple cells. It also provides techniques of Coordinated Scheduling (CS) with Dynamic Point Blanking (DPB) to optimize the radio resource distribution and determine which eNBs should blank transmission in certain RBs or frequency sub-bands so as to limit the interference to neighboring cells [8].

The introduction of small cells has raised a new challenge to HetNets especially the user association, which is to define a set of rules for optimally attaching users to different eNBs in the network. Attaching a user to the optimal eNB can significantly improve its throughput as well as the overall network utillity. In conventional homogeneous LTE networks, user association is based on the strongest downlink received signal. This policy is not suitable to HetNets, where small cells and macro cells operate at different transmission power. To perform the best user association in HetNets, different association algorithms have been proposed, see for example [17]. Cell range extension by applying Cell Individual Offset has been proposed by 3GPP in Release 10 [13]. In user association, the power strength received at a user from small cell would be artificially added by an offset, in order to offload macro cells and to ensure that more users would attach to small cells.

By assuming that all cells use all the spectrum (i.e., reuse-1), [18] investigated a joint user association and intra-cell resource allocation optimization problem. The joint optimization of Almost Blank Sub-frames and user association was studied in [10] and [11], where each macro BS is assumed to have the same muted sub-frames. The principles of user association using CIO and the inter cell interference management have been proposed in 3GPP Releases. However, the standards do not specify how to implement these mechanisms and how to set optimal parameters in different network configurations.

Generally speaking, today's solutions are usually limited in their scope due to the inherent complexity of the optimization problem. Previous studies often either consider disjoint optimization of user association and interference management or consider specific network utility functions for facilitating optimization algorithms using convexity and specific implementation constraints. To the best of our knowledge, there has not been, so far, efficient and practical solution able to handle all these requirements with high deployment flexibility.

Our proposed framework explores the idea of a central coordinator that gathers some information concerning the eNBs and the users in the system to determine optimal parameters. This idea is well aligned with the emerging technology for designing and managing mobile networks through Software Defined Wireless Networking (SDWN). This new paradigm, simplifies network management by decoupling the control plane and data plane and enabling operators to have a complete control over the network from a centralized point. For example, in [20], we design a SDWN controller based on OpenDayLight and we validate our framework with a sub optimization algorithm, handling $\mathrm{CIO}$ and ABS, deployed as a north bound (NB) application.

\section{System Model AND Problem Formulation}

We consider an LTE cellular network composed of $K$ cells: $M$ macro cells and $N$ small cells, where $N \geq 0$, to model both homogeneous and heterogeneous networks. Each base station $k$ has $S$ sub-frames in the time domain and $R$ resource blocks in the frequency domain, respectively. The duration of all subframes is the same and the bandwidth of all RBs is also a constant; for example according to 3GPP LTE standard [16], they are $1 \mathrm{~ms}$ and $180 \mathrm{kHz}$, respectively.

\section{A. System Model}

In our LTE frequency sub-band coordinated scheduling system, all the RBs are first grouped into $F$ frequency subbands, where each sub-band consists in a number $R_{f}$ of RBs, $f=1,2, \ldots, F$. The bandwidth of each sub-band is thus given by $R_{f} B$, where $B=180 \mathrm{kHz}$. In the same manner, we regroup the $S$ sub-frames into $T$ time slots, where each slot consists in a number $S_{t}$ of sub-frames, $t=1,2, \ldots, T$. The duration of each slot $t$ is equal to $S_{t} \mathrm{~ms}$.

Here, we only consider downlink (DL). The LTE transmissions in each cell are synchronized such that there is no intracell interference. However, there exists inter-cell interference, i.e., a transmission from a cell will cause interference to other cells which reuse the same RBs at the same time.

We will use $P_{k, f, t}$ to denote the power allocated by cell $k$ to frequency sub-band $f$ at time slot $t$. Note that $P_{k, f, t}$ is in discrete value. We also define vector $P_{k}:=\left(P_{k, 1,1}, P_{k, 2,1}, \ldots\right.$, $\left.P_{k, F, 1}, P_{k, 1,2}, P_{k, 2,2}, \ldots, P_{k, F, 2}, \ldots, P_{k, F, T}\right)$. The total power of a cell $k$ at time slot $t$ is limited by a maximum value $P_{k}^{\max }$ such that

$$
\sum_{f \in F} P_{k, f, t} \leq P_{k}^{\max }, \forall t \in T, \forall k \in K
$$


Denote $\mathcal{U}_{k}$ as the set of users who are associated with cell $k$ and $\mathcal{U}$ as the set of all the users in the network, respectively. We use binary variable $Q_{u, k}$ to indicate whether a user $u$ is in $\mathcal{U}_{k}$ or not. Here, each user can be served by only one cell such that

$$
\sum_{k \in K} Q_{u, k}=1, \forall u \in \mathcal{U}
$$

Let $N_{k}$ be the set of neighboring eNBs of eNB $k$. The actual definition of this set will be presented in the next section. We will use $\bar{O}_{k, k^{\prime}}$ to denote the CIO in $\mathrm{dB}$ from cell $k$ to its neighboring cell $k^{\prime}$. The possible CIO values between each two neighboring cells range from $-24 \mathrm{~dB}$ to $24 \mathrm{~dB}$ but reasonable values often are in $10 \mathrm{~dB}$ range. Generally, some reciprocity is required in the CIO, i.e., $\bar{O}_{k, k^{\prime}}=-\bar{O}_{k^{\prime}, k}$. We define vector $\bar{O}_{k}:=\left(\bar{O}_{k, 1}, \bar{O}_{k, 2}, \ldots, \bar{O}_{k, N_{k}}\right)$.

We use notation $s_{k}:=\left(P_{k}, \bar{O}_{k}\right)$ to represent the power and CIO settings for each cell and $\mathbf{s}:=\left(s_{1}, s_{2}, \ldots, s_{K}\right)$ as the network profile, respectively. Given a network state $s \in \mathbf{S}$, where $s$ is a configuration of $P_{k}$ 's and $\bar{O}_{k}$ 's of the $K$ cells, we aim to determine the optimal values of $P_{k}$ 's and $\bar{O}_{k}$ 's for maximizing the network or total utility $U(s)$ :

$$
U(s)=\sum_{k \in K} U_{k}(s)
$$

where $U_{k}$ is the utility for cell $k$, which is usually determined by the achievable throughputs of users of cell $k$. If one may consider proportional fairness network utility, then we have

$$
U_{k}(s)=\sum_{u \in \mathcal{U}_{k}} \log \left(r_{u}(s)\right)
$$

where $r_{u}(s)$ denotes the throughput of user $u$. In the coming discussion, we may write $r_{u}$ instead of $r_{u}(s)$ for notation simplicity.

Under the Additive White Gaussian Noise (AWGN) model by Shannon-Hartley theorem, the throughput (in bits $/ \mathrm{s} / \mathrm{Hz}$ ) achievable by user $u$ can be expressed as

$$
r_{u}=W \log \left(1+\operatorname{SINR}_{u}(s)\right)
$$

where $W$ is the bandwidth of the channel (in $\mathrm{Hz}$ ) and the SINR of user $u$ when served by cell $k$ which transmits over frequency sub-band $f$ during time slot $t$ is expressible as

$$
\operatorname{SINR}_{u, k, f, t}=\frac{P_{k, f, t} G_{u, k, f, t}}{\eta_{u}+\sum_{l \neq k} P_{l, f, t} G_{u, l, f, t}}
$$

with $\eta_{u}$ representing the thermal background noise and $G_{u, k, f, t}$ denoting the link gain from cell $k$ to user $u$ over frequency sub-band $f$ during slot $t$.

\section{B. Problem Setup}

For generality, one can re-write (4) as

$$
U_{k}(s)=\sum_{u \in \mathcal{U}_{k}} C\left(\operatorname{SINR}_{u}(s)\right)
$$

where $C(\cdot)$ is a utility function.

Let $\tau_{u, k, f, t}$ be the number of RBs out of $R_{f}$ of frequency sub-band $f$ granted by cell $k$ to user $u$ during slot time $t$.
Clearly, in the resource allocation and transmission scheduling at each eNB, we will have the following constraints

$$
\sum_{t=1}^{T} \sum_{f=1}^{F} \sum_{u \in \mathcal{U}_{k}} \tau_{u, k, f, t} \leq R \times S
$$

where $R \times S$ is the total amount of resource blocks in the system during $S$ sub-frames, and

$$
\sum_{u \in \mathcal{U}_{k}} \tau_{u, k, f, t} \leq R_{f} \times S_{t}
$$

for each frequency sub-band and time slot.

To address the power allocation optimization problem for LTE network management, we will use long-term statistics in both the frequency and time domains such that $\operatorname{SINR}_{u, k, f, t}$ is a measure of averaged SINR of user $u$ over RBs in the frequency sub-band $f$ and during time slot $t$. We will therefore write $\operatorname{SINR}_{u, k, f, t}$ after averaging the channel variations which may be due to fast fading and frequency selectiveness. This is also to reduce the optimization problem and its dimension.

As a result, the total throughput received by a user $u$ can be measured by

$$
r_{u}=Q_{u, k} \sum_{t=1}^{T} \sum_{f=1}^{F}\left[\tau_{u, k, f, t} \times B \log \left(1+\operatorname{SINR}_{u, k, f, t}\right)\right] .
$$

The RB time scheduling can be another optimization procedure or one may use a simple LTE downlink scheduler.

The user association problem using CIO can be addressed as follows:

$Q_{u, k}= \begin{cases}1 & \text { if } \forall k^{\prime} \in N_{k}, \overline{R S R P}_{u, k}>\overline{R S R P}_{u, k^{\prime}}+\bar{O}_{k, k^{\prime}} \\ 0 & \text { otherwise. }\end{cases}$

where $\overline{R S R P}_{u, k}$ and $\overline{R S R P}_{u, k^{\prime}}$ are the RSRP measurements in $\mathrm{dB}$ of user $u$ corresponding to the cells $k$ and $k^{\prime}$ and can be approximated in this manner:

$$
\left\{\begin{array}{l}
\overline{R S R P}_{u, k}=\bar{P}_{k}^{\max }+\bar{G}_{u, k} \\
\overline{R S R P}_{u, k^{\prime}}=\bar{P}_{k^{\prime}}^{\max }+\bar{G}_{u, k^{\prime}}
\end{array}\right.
$$

where $\bar{G}_{u, k}$ and $\bar{G}_{u, k^{\prime}}$ are the averaged link gain from cell $k$ and $k^{\prime}$ respectively to user $u$ over all the bandwidth and over a certain time duration, expressed in $\mathrm{dB}$. Notice that for notation simplicity we simply $\bar{G}_{u, k}$ and $\bar{G}_{u, k^{\prime}}$ instead of $\bar{G}_{u, k}^{(\mathrm{dB})}$ and $\bar{G}_{u, k^{\prime}}^{(\mathrm{dB})}$. Similarly, $\bar{P}_{k}^{\max }$ and $\bar{P}_{k^{\prime}}^{\max }$ are the maximum power transmission in $\mathrm{dB}$ of the cells $k$ and $k^{\prime}$, respectively.

To summarize, our goal is to maximize the network utility (3) with the following decision variables:

- CIO $O_{k}=\left(O_{k, 1}, O_{k, 2}, \ldots, O_{k, N_{k}}\right)$ that defines the offsets to be added to RSRP measurements and affects users' attachment to their neighboring cells,

- Power vector over frequency and time domain $P_{k}=$ $\left(P_{k, 1,1}, P_{k, 2,1}, \ldots, P_{k, F, 1}, P_{k, 1,2}, \ldots, P_{k, F, 2}, \ldots, P_{k, F, T}\right)$ that defines the power allocation by each cell over frequency sub-band and time slot. 


\section{Special Cases}

From our generalized problem, we can specify certain special cases, with simple modifications or restrictions of the optimization problem parameters.

- Disjoint Frequency Sub-band Optimization: Determine for each cell $k$ which frequency sub-bands it is allowed to use. By taking $T$ equal to 1 , we eliminate the time dimension of the problem and consider that we have the same power pattern over all sub-frames. The power vector is then defined by $P_{k}=\left(P_{k, 1,1}, P_{k, 2,1}, \ldots, P_{k, F, 1}\right)$. We restrict the possible power levels per RB to either 0 , i.e., the frequency resource cannot be used by the cell $k$, or the maximum power per RB, which is equal to $P_{k}^{\max }$ divided by the total number of RBs. By playing with the number of frequency sub-bands $F$ and the number of sub-frames in each sub-band, we obtain different frequency sub-band optimization problems. One extreme case is to take $F$ equal to $S$, i.e., each frequency sub-band is composed of one RB. The user association can be done according to the usual policy of strongest received signal by restricting the possible values of CIO to 0 .

- Disjoint Power Level Optimization: Determine for each cell $k$ the power value to be used over each frequency sub-band. The time dimension is not taken into account and the number of frequency sub-bands and their size are fixed. The optimization is accomplished by playing with the possible power values per RB. Generally, the maximum transmission power of the cell $k$ is divided equally over the RBs, and each resource has a maximum power level defined as $\frac{P_{k}^{\max }}{R}$. With the power level optimization, it is possible to boost the power on some resources or lower it.

- Time Optimization: Determines for each cell $k$ a time pattern over all the bandwidth, i.e., during which slots the BS can transmit. 3GPP Release 10 has defined Almost Blank Sub-frames to mute macro cells during certain slots and minimize their interference, while small cells can transmit all the time. The ABS optimization problem can be expressed by adding some restrictions to our generalized problem formulation. We group all RBs into one frequency sub-band, i.e., $F=1$. The power pattern vector is then defined by $P_{k}=\left(P_{k, 1,1}, P_{k, 1,2}, \ldots, P_{k, 1, T}\right), \forall k \in$ $M$ and $P_{k}=P_{k}^{\max } \forall k \in S$.

- User Association Optimization: Determine the policy to attach each user to a cell. It is possible to apply existing policies already used by the operator and to use the resulting attachments directly in the rest of the optimization. When adopting the CIO optimization to define the user association, a general formulation of the problem is to take different CIO values between each two neighboring cells. However, the problem can be restricted by choosing a unique $\mathrm{CIO}$ value for each cell $k$ towards all its neighboring cells, we got then $\bar{O}_{k}=$ constant.

- Utility Function Maximization: The use of the proportional fair is just an example of one possible network utility to be used in the optimization. Other parameters can be taken into account in the cost function such as other QoS criteria and minimum rate requirement.

\section{Proposed Scheme}

In this section, we present an approach using potential games that achieve an optimal solution for the inter-cell interference coordination and frequency sub-band reuse and power allocation optimization. We model the problem as a non-cooperative game where the eNBs are players and we introduce the potential game approach that relies on approximating the non-cooperative games with potential games.

\section{A. Theoretical Background}

Let eNBs periodically broadcast pilot signals of a priory fixed power. We formulate our objective function into a potential game by designing cost functions of eNBs and their neighbours. To begin with, we define the set of all neighbours of an eNB $k$, named $N_{k}$, as follows: $N_{k}$ is the set of eNBs such that the received pilot signal power at user $i$ from an eNB in $N_{k}$ is greater than a certain threshold, say $\theta$.

In our development we use the following property: For any eNB pair $(k, j), j \in N_{k} \Leftrightarrow k \in N_{j}$. We use $N_{k}^{+}$to denote the set of eNBs including eNB $k$ and its neighbors, i.e., $N_{k}^{+}=$ $k \cup N_{k}$.

We model the resource allocation problem as the following non-cooperative game:

Players: The eNBs are the players. Let's denote the set of players by $\mathcal{K}$.

Strategies: For eNB $k$, a strategy is a tuple $s_{k}=\left(P_{k}, \bar{O}_{k}\right)$ and its strategy set is $\mathcal{S}_{k}:=\mathcal{P}_{k} \times \overline{\mathcal{O}}_{k}$, where $\mathcal{P}_{k}$ and $\overline{\mathcal{O}}_{k}$ are the spaces of $P_{k}$ and $\bar{O}_{k}$, respectively. A joint strategy $\mathbf{s}=$ $\left(s_{k}, k \in \mathcal{K}\right)$ belongs to the joint strategy space $\mathcal{S}:=\times_{k \in \mathcal{K}} \mathcal{S}_{k}$.

Payoffs: eNB $k$ 's payoff function $M_{k}: \mathcal{S} \rightarrow \mathbb{R}$ is defined as

$$
M_{k}(s):=\sum_{j \in N_{k}^{+}} \tilde{U}_{j}(s)
$$

where the function $\tilde{U}_{j}(s)$ is defined as in (7) but the SINR defined in (6) is replaced by

$$
\operatorname{SINR}_{u, k, f, t}=\frac{P_{k, f, t} G_{u, k, f, t}}{\eta_{u}+\sum_{l \in N_{k}} P_{l, f, t} G_{u, l, f, t}} .
$$

In the following, we refer to this game as the strategic form game $\left(\mathcal{K},\left(\mathcal{S}_{k}, k \in \mathcal{K}\right),\left(M_{k}, k \in \mathcal{K}\right)\right)$. With this modification, each eNB needs only to know some specific information from its neighbors $N_{i}$. Hence, the accuracy of the potential game approach depends on the value of the sensitivity threshold $\theta$ since the size of the neighborhood increases when the value of $\theta$ decreases. With $\theta=0$, the Nash equilibrium of the potential game coincides with the optimal solution of the utility (3).

In the following result, we prove that non-cooperative game is a potential game, which is known to have interesting properties, such as existence of a Nash equilibrium and convergence of best-response algorithm to this equilibrium in a finite number of steps, for the completeness.

Theorem 1. The finite strategic form game $\left(\mathcal{K},\left(\mathcal{S}_{k}, k \in\right.\right.$ $\left.\mathcal{K}),\left(M_{k}, k \in \mathcal{K}\right)\right)$ is a potential game and thus admits finite improvement path (FIP [19]). 
Proof: Let players use a strategy $\mathbf{s}=\left(s_{k}, k \in \mathcal{K}\right)$. Consider a player $k$ and assume that it changes its strategy from $s_{k}$ to $s_{k}^{\prime}$. We have

$$
\begin{aligned}
& U\left(s_{k}^{\prime}, \mathbf{s}_{-k}\right)-U(\mathbf{s})=\sum_{j \in \mathcal{K}}\left(U_{j}\left(s_{k}^{\prime}, \mathbf{s}_{-k}\right)-U_{j}(\mathbf{s})\right) \\
& \stackrel{(*)}{=} \sum_{j \in N_{k}^{+}}\left(U_{j}\left(s_{k}^{\prime}, \mathbf{s}_{-k}\right)-U_{j}(\mathbf{s})\right)+\sum_{j \notin N_{k}^{+}}\left(U_{j}\left(s_{k}^{\prime}, \mathbf{s}_{-k}\right)-U_{j}(\mathbf{s})\right) \\
& =\sum_{j \in N_{k}^{+}}\left(U_{j}\left(s_{k}^{\prime}, \mathbf{s}_{-k}\right)-U_{j}(\mathbf{s})\right) \\
& =M_{k}\left(s_{k}^{\prime}, \mathbf{s}_{-k}\right)-M_{k}(\mathbf{s})
\end{aligned}
$$

where $(*)$ is due to the fact that $\forall j \notin N_{k}^{+}, k \notin N_{j}^{+}$and thus $U_{j}(\mathbf{s})$ is independent of eNB $k$ 's strategy. Therefore, the function $U(\cdot)$ is a potential function for the game $\left(\mathcal{K},\left(\mathcal{S}_{k}, k \in\right.\right.$ $\left.\mathcal{K}),\left(M_{k}, k \in \mathcal{K}\right)\right)$. This implies FIP property.

\section{B. Proposed Solution}

In the following, we will describe the algorithm and its operation in performing user association and frequency/time resource allocation via power patterns optimization for LTE cellular networks. This solution based on game theory is an extension to our work on coordinated scheduling via frequency and power allocation optimization presented in [3]. Fig. 1 shows the design where a coordinator optimizes CIO values, virtually attaches the users to corresponding cells, performs a dynamic resource distribution, virtually schedules the users in the network, and computes a utility function. Then, it sends the optimal parameters (power allocation pattern and CIO values) to each cell. The CIO values are added to RSRP measurements and this impacts the user association and handover decision. Then, each local eNB scheduler allocates its provided RBs according to its scheduling policy and uses the power settings determined from the optimizer.

1) Step 1 - Data Collection: Each user (UE) reports to its serving eNB long term statistics, such as Channel Quality Indication (CQI) and Reference Signal Received Power (RSRP). These measurements are processed by the eNB to group the users in pools having similar channel quality, then they are sent via $\mathrm{S} 1$ protocol to the coordinator. Various examples of UE grouping are presented in Fig. 2, for illustration. The grouping allows to limit the data exchange between the eNBs and the central entity that is in charge of the optimization. In this way, the eNB keeps a precise and real time knowledge of its attached users. Meanwhile, the coordinator keeps a global view over the cells in the network with access only to longterm statistics that are averaged both in spatial dimension (by the UE grouping) and in time dimension (between two update messages).

2) Step 2-Optimization: At the end of collection step, the coordinator has a database containing the needed information concerning the state of the network. Working on this database, it performs an optimization to deliver the optimal parameters: $\mathrm{CIO}$ value and transmission power pattern for each cell. Fig. 1 shows the performed iterations.

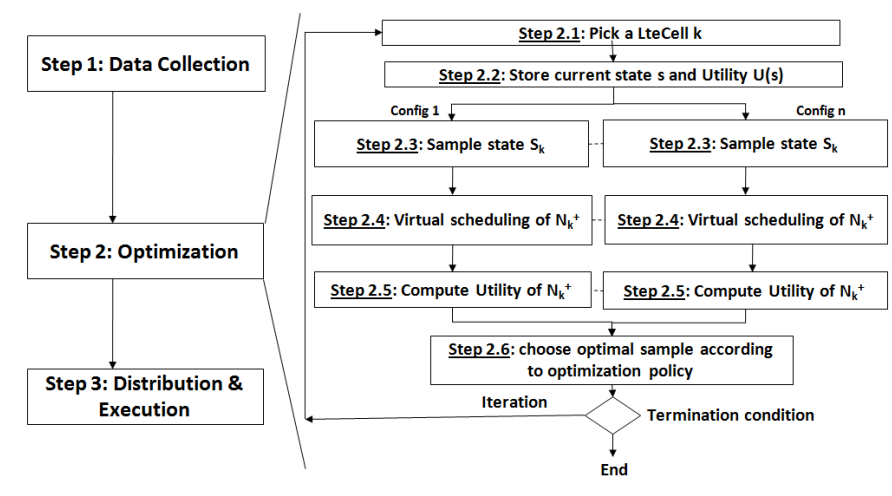

Fig. 1. Framework overview.

a) Steps $2.1 \& 2.2$ - Choose a cell and store initial state and utility: The coordinator picks up a cell randomly. It stores the initial network state, which refers to the CIO and power setting of each cell in the system. The coordinator computes the initial global utility which indicates the network performance.

b) Step 2.3 - Sampling: This step consists in sampling the couple $\left(P_{k}, \bar{O}_{k}\right)$ for the selected cell. For each neighboring cell, we attribute a CIO value, which can be positive or negative. The total number of RBs depends on the system, e.g., given $5 \mathrm{MHz}$ and $10 \mathrm{MHz}$, there will be 25 and 50 RBs, respectively. For simplicity and practical use, we limit the configuration options in the optimizer such that the RBs are grouped into equally sized sub-bands or approximately. Fig. 3 shows the details in the case of $10 \mathrm{MHz}$. In the same manner, we regroup the $S$ subframes into $T$ equally sized slots. One resource element is defined by the couple of frequency sub-band and time slot. Sampling $P_{k}$ consists on allocating a transmission power over each frequency sub-bands and time slot.

Note that the sampling of states is performed among the admissible combinations of power settings and CIO values. In practice, the sampling of states can be done in parallel. Given $N_{k}$ neighboring cells and $I$ possible offset values, we have $I^{N_{k}}$ possible samples for $\bar{O}_{k}$. Given $F$ frequency sub-bands, $T$ time slots and $Y$ power levels per RB, we have $Y^{F \times T}$ samples for the power patterns. This implies that one will have $I^{N_{k}} Y^{F \times T}$ cases to be sampled for the cell selected in Step 2.1. However, some combinations can be easily discarded with respect to some constraints such as maximum power. As mentioned, performing disjoint optimization is also possible in our framework. The complexity of the sampling can be then reduced. For instance, in ABS optimization, only macro cells are concerned with the muting.

c) Step 2.4 - Virtual handover: For each sampled case where the CIO has been changed, we perform a virtual handover by calling the ueGroupAttach function. This function tests if the user group would make a handover to a neighboring cell due to the change in the CIO. It compares the RSRP measurements to the serving and neighboring cells after adding the new sampled CIO value, and virtually changes the user association accordingly. 


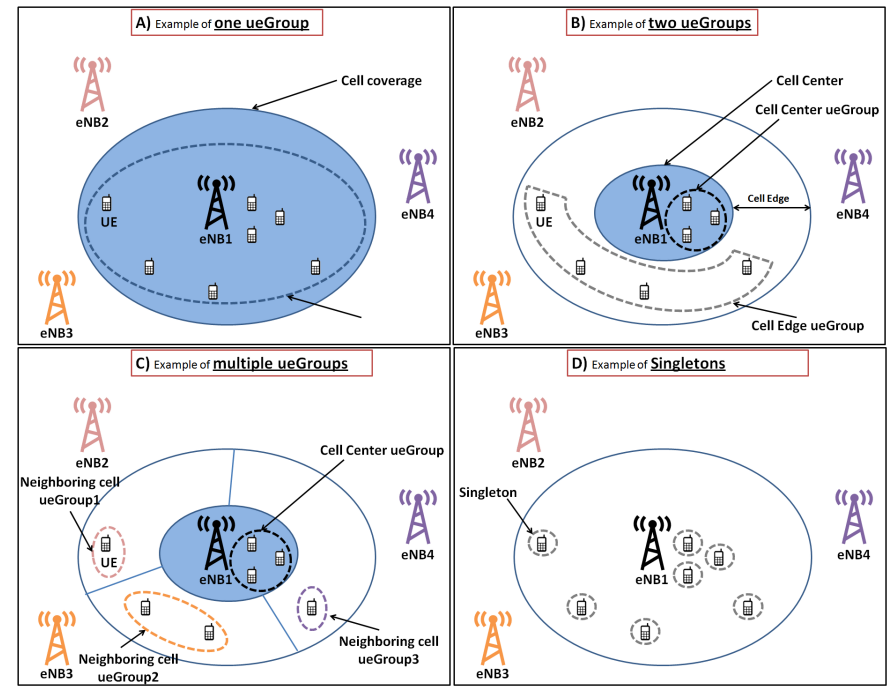

Fig. 2. Example of various UE grouping policies.

d) Step $2.5 \& 2.6$ - Virtual scheduling \& utility computation: For each sampled configuration, the optimizer calls this function to render the scheduling performed by each eNB (the one being selected in Step 2.1 and its neighboring eNBs) in order to estimate the expected performance (resulting bit rates). Several options are available such as proportional fairness, absolute fairness (max-min), sum rate maximization, etc. For each eNB and its UEs, we adopt the well-known Proportional Fair Scheduling (PFS) algorithm used in today's LTE [2]: the PFS will serve a UE $u_{m}$ when its instantaneous channel quality is the highest according to

$$
u_{m}=\underset{u \in \mathcal{U}_{k}}{\arg \max } \frac{R_{u}(m, t)}{\bar{R}_{u}(t)}
$$

where $\bar{R}_{u}(t)$ denotes the experienced average throughput of user $u$ at time $t$ and $R_{u}(m, t)$ is the achievable rate by user $u$ if it may get served by the RB $m$.

To compute the throughput utility for each cell, we propose a virtual scheduling approaching to the PFS. These inputs are needed:

- $S$ sub-frames grouped in $T$ time slots. For simulation purposes, $S$ is equal to 80 .

- $F$ sub-bands and $R_{f}$ number of RBs in each frequency sub-band.

- The averaged spectral efficiency $s e_{u, f, t}$ for each user $u$ over each sub-band $f$ during time slot $t$ is derived from $\operatorname{SINR}_{u, k, f, t}$.

After calling the virtual scheduling function for the selected cell and its neighboring cells, the coordinator computes the utility function based on the resulting achievable rates $r_{u}$ 's. Note that indeed the proposed framework can support various optimization tools and utility definitions, depending on operator's strategy. It is not limited to proportional fairness utility.

e) Step 2.6 - Choosing optimal sample: After sampling all the possible states of the chosen cell and computing their corresponding utility values, the coordinator chooses the best configuration for maximum value, i.e., best response. As
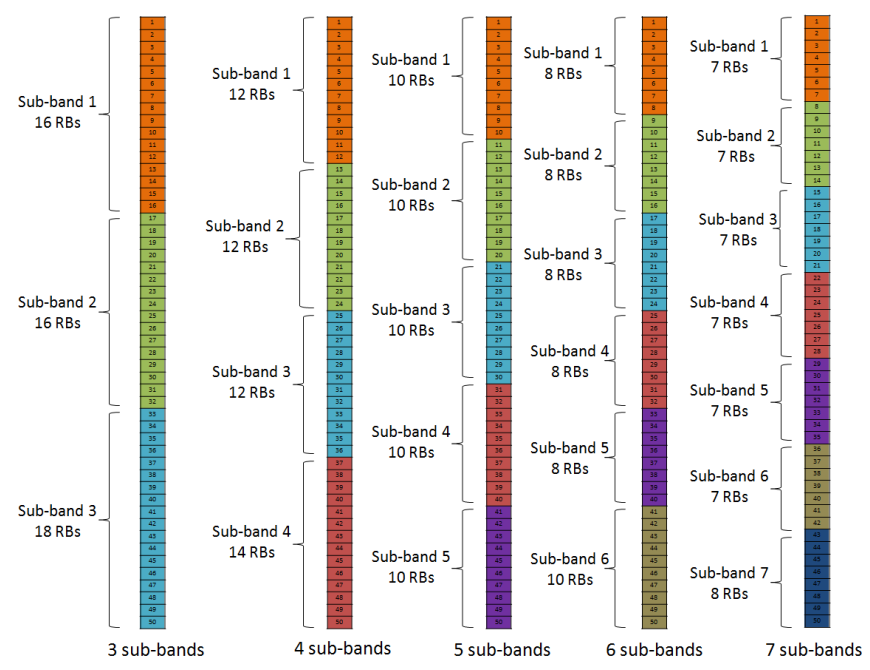

Fig. 3. Frequency bandwidth are divided into sub-bands.

TABLE I

SIMULATION ENVIRONMENT

\begin{tabular}{|c|c|}
\hline Radio & $\begin{array}{c}\text { LTE Rel. 8, SISO, 10MHz, 3GPP recommended pathloss } \\
\text { and shadow fading models }\end{array}$ \\
\hline Topolgy & $\begin{array}{c}\text { Hexagonal } 1 \text { ring, ISD 500m, } 3 \text { cells per macro site } \\
30 \text { small cells (SC) at fixed location } 0.5 \text { ISD } \\
\text { Antenna (MC/SC): Kathrein /omnidirectional } \\
\text { Max Power of MC and SC: } 40 \mathrm{~W}, 1 \mathrm{~W}\end{array}$ \\
\hline $\begin{array}{l}\text { Mobile } \\
\text { users }\end{array}$ & $\begin{array}{c}\text { Scenario 1: } 25 \text { users/MC, total of } 525 \text { users } \\
\text { Scenario 2: } 25 \text { users/MC, } 10 \text { users/SC, total of } 825 \text { users } \\
\text { No mobility, full buffer model }\end{array}$ \\
\hline $\begin{array}{l}\text { Optimization } \\
\text { sampling }\end{array}$ & $\begin{array}{c}F \in\{1,3,4,5,6,7\}, T \in\{1,4,8\} \\
\text { MC_Power_per_RB } \in\{0,0.4,0.8,1.2,1.6\} \\
\text { SC_Power_per_RB } \in\{0,0.01,0.02,0.03,0.04\} \\
\bar{O}_{k, k^{\prime}} \in\{0,5,-5\}\end{array}$ \\
\hline
\end{tabular}

previously discussed, the best response update is guaranteed to converge to a Nash equilibrium through a finite number of iterations.

3) Distribution \& Execution: After the optimization, the coordinator sends the optimized setting to each eNB. The optimized CIO values are added to RSRP measurements to trigger possible handovers. The local schedulers allocate their provided RBs with respect to their power level patterns over time and frequency dimension, as advised by the coordinator.

\section{Performances and Simulation Results}

\section{A. Simulation Setup \& Metrics}

To emulate the LTE network, we used a MATLAB-based LTE-compliant simulator developed by the TU Wien's Institute of Telecommunications [21]. This tool generates the eNBs, the pathloss model, the users, etc., and allows to measure the performance with various KPIs. Table I gives the simulation parameters. To evaluate the performance of the proposed framework, we measured: 
TABLE II

DisJoINT FREQUENCY SUB-BAND OPTIMIZATION RESULT - ONLY MACROS

\begin{tabular}{|c|c|c|c|c|c|c|}
\hline$T=1$ & $\begin{array}{c}\text { Static } \\
\text { reuse-1 }\end{array}$ & $F=3$ & $F=4$ & $F=5$ & $F=6$ & $F=7$ \\
\hline $\begin{array}{l}\text { Avg-user-Th } \\
\text { (kbps) }\end{array}$ & 301.7 & 316.2 & 328.7 & 335.6 & 342.4 & 345.6 \\
\hline Gain \% & - & $+4.8 \%$ & $+9 \%$ & $+11.2 \%$ & $+13.5 \%$ & $+14.6 \%$ \\
\hline $\begin{array}{l}\text { Cell-edge-Th } \\
\text { (kbps) }\end{array}$ & 38.9 & 43.7 & 54.7 & 55 & 54.8 & 56 \\
\hline Gain \% & - & $+12.4 \%$ & $+\mathbf{4 0 . 8 \%}$ & $+41.5 \%$ & $+41.1 \%$ & $+44.4 \%$ \\
\hline $\begin{array}{c}\text { Avg-EE } \\
\text { (bits/joule) }\end{array}$ & 190.7 & 228 & 263.2 & 258.4 & 258 & 258.7 \\
\hline Gain \% & - & $+19.6 \%$ & $+38 \%$ & $+35.5 \%$ & $+35.3 \%$ & $+35.7 \%$ \\
\hline $\begin{array}{l}\text { Cell-edge-EE } \\
\text { (bits/joule) }\end{array}$ & 20.5 & 25.7 & 32.2 & 30.1 & 29.5 & 30.2 \\
\hline Gain \% & - & $+25 \%$ & $+57 \%$ & $+47 \%$ & $+44 \%$ & $+47 \%$ \\
\hline
\end{tabular}

- Average user throughput (kbps)

- Cell edge user throughput (kbps): defined as the 5th percentile point of the CDF of user throughput. It represents the maximum throughput of the 5\% users experiencing worst data rate in the network.

- Average energy efficiency (bits/joule): defined as the ratio of total amount data delivered to all the users and the total power consumed in the network.

- Cell edge energy efficiency (bits/joule): defined as the ratio of bits delivered to cell edge users and the amount of power consumed to transmit these data.

In the following, The first scenario, called 'macros only' refers to the case where we simulate 525 users that are only initially attached to macro cells. In the second scenario 'HetNets', besides the users in the coverage of macro cells as in first scenario, we simulate 300 more users that are associated to small cells.

\section{B. Disjoint Frequency Sub-band Optimization}

As shown in Fig. 3, we consider frequency sub-bands with various possible sizes and compare to the case of frequency reuse- 1 for the following two scenarios: (i) macros only and (ii) HetNets.

For each configuration, 20 simulation sets were run with different users distributions.

The results of frequency sub-bands optimization are presented in Table II and III. The gains are computed against the case of static reuse- 1 system.

We obtain gains of:

- about $14 \%$ in average user throughput which increases from $301 \mathrm{kbps}$ in the case of static reuse-1 to 345 kbps using the 7 sub-bands dynamic optimization in first scenario - only macro cells. In HetNets, the gain is even higher due to the presence of small cells in the coverage
TABLE III

Disjoint Frequency Sub-BAnd Optimization Result - HetNets

\begin{tabular}{|c|c|c|c|c|c|c|}
\hline$T=1$ & $\begin{array}{c}\text { Static } \\
\text { reuse-1 }\end{array}$ & $F=3$ & $F=4$ & $F=5$ & $F=6$ & $F=7$ \\
\hline $\begin{array}{l}\text { Avg-user-Th } \\
\quad(\mathrm{kbps})\end{array}$ & 381.4 & 458.4 & 464 & 462.4 & 474.3 & 477 \\
\hline Gain \% & - & $+20.1 \%$ & $+21.6 \%$ & $+21.2 \%$ & $+24.3 \%$ & $+25 \%$ \\
\hline $\begin{array}{l}\text { Cell-edge-Th } \\
\quad \text { (kbps) }\end{array}$ & 40.4 & 49.5 & 54.5 & 55.4 & 57.9 & 58.5 \\
\hline Gain \% & - & $+22.6 \%$ & $+34.9 \%$ & $+37 \%$ & $+43.2 \%$ & $+\mathbf{4 4 . 8 \%}$ \\
\hline $\begin{array}{c}\text { Avg-EE } \\
\text { (bits/joule) }\end{array}$ & 365.6 & 583.1 & 583.1 & 561.8 & 588.2 & 588.2 \\
\hline Gain \% & - & $+59.5 \%$ & $+59.5 \%$ & $+53.6 \%$ & $+60.9 \%$ & $+60.9 \%$ \\
\hline $\begin{array}{c}\text { Cell-edge-EE } \\
\text { (bits/joule) }\end{array}$ & 20.5 & 31.1 & 32.2 & 31.8 & 33.5 & 33.3 \\
\hline Gain \% & - & $+51.2 \%$ & $+56.7 \%$ & $+54.7 \%$ & $+63.2 \%$ & $+62 \%$ \\
\hline
\end{tabular}

of macros. We observe a gain of $25 \%$ in average user throughput.

- about $40 \%$ in cell edge throughput for both the macros and HetNets scenarios. This significant improvement is due to a better inter-cell interference mitigation. Using a dynamic frequency sub-band pattern, neighboring cells schedule their users in different sub-bands which limits strongly the interference especially for cell edge users. Thus, for the macros scenario, the cell edge throughput goes from $39 \mathrm{kbps}$ to $56 \mathrm{kbps}$, using the static reuse- 1 and the 7 sub-bands dynamic scheme respectively. In HetNets configuration, the cell edge users throughput increases from $40 \mathrm{kbps}$ to $58 \mathrm{kbps}$.

- about $35 \%$ and $60 \%$ in average energy efficiency for macros and HetNets scenarios, respectively. As we do not use power boosting in this frequency sub-band optimization, the power transmission on one RB is either zero or equal to the maximum power per RB, i.e., $0.8 \mathrm{~W}$ for macro cell and $0.02 \mathrm{~W}$ for small cell. On one hand, when some sub-bands are muted, the sum of powers on all other sub-bands is inferior to the maximum of power of the cell, thus leads to energy saving. On the other hand, muting some resources on one cell permits its neighboring cells to have better spectral efficiency on the muted sub-band and then to transmit more data to their attached users. We also see the increase of the average energy efficiency from the macros only scenario to HetNets scenario.

- about $50 \%$ and $60 \%$ in cell edge energy efficiency for macros and HetNets scenarios, respectively.

Regarding cell edge throughput, we observe significant gains from the 3 sub-bands to the 4 sub-bands case and then the gain slows down from the 4 sub-bands to the 7 subbands configurations. This can be explained by the trade-off between throughput loss when muting some resources for one cell and interference decrease for the neighboring cells which would improve the channel quality especially for cell edge 

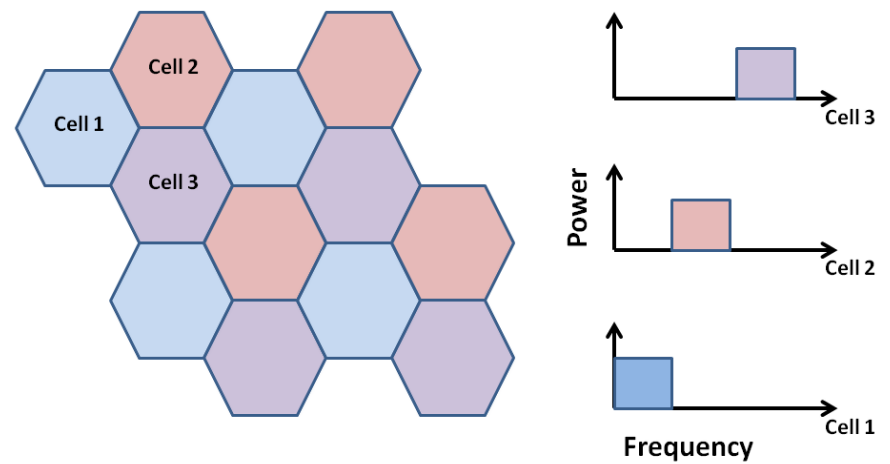

Fig. 4. Frequency Reuse 3.

users. In the case of the 3 sub-bands configuration, as a result of the optimization, at most only 1 sub-band of 16 or 18 RBs is muted per cell, which corresponds to a third of the available bandwidth. In the other sub-bands configuration, it could happen that 2 or even more sub-bands are muted for some cells. In general, the optimized size of muted sub-bands does not exceed the half of the bandwidth.

In macros only scenario, we observe similar performance trends in the average throughput, average energy efficiency, and cell edge energy efficiency. They converge to a maximum value starting from the 4 sub-bands case. In HetNets, this convergence is more or less obtained starting from the 4 subbands case.

To further evaluate the gain of our proposed dynamic frequency sub-bands optimization, we conducted extra simulation sets using static frequency reuse of 3. As shown in Fig. 4, this approach consists in dividing the frequency into 3 sub-bands and only one band of the frequency is allocated to each cell in the macro site. The same frequency is reused every 3 cells. Since the resources are under utilized in this configuration, it is expected to have lower average user throughput in the system. In the first scenario with homogeneous networks, the average user throughput only reachs $227 \mathrm{kbps}$. Using our 7 sub-band optimization algorithm for example, the average throughput can be improved by above 50\% againt the static frequency reuse- 3 configuration. Compared to the static reuse1 scenario, cell edge users have better signal quality since the inter-cell interference is lowered. In the macros only scenario, cell edge throughput is about $50 \mathrm{kbps}$, which represents a gain of $30 \%$ compared to reuse- 1 . Our proposed optimization delivers better cell edge performance than the frequency reuse of 3.

We can draw the following summary:

- Dynamic frequency sub-bands optimization always outperforms static frequency reuse-1 scheme in terms of average and cell edge throughput and energy efficiency.

- The gains are increasing with the number of frequency sub-bands.

- For macros only scenario, the optimal frequency patterns are obtained at the 4 sub-band optimization. A significant gap is observed from the 3 sub-bands to the 4 subbands case. For HetNets scenario, very good results are obtained since the first optimization configuration with
TABLE IV

Disjoint Power Levels Optimization Result - Only Macros

\begin{tabular}{|c||c|c|c|}
\hline $\boldsymbol{F = 3 ,} \boldsymbol{T = 1}$ & $\begin{array}{c}\mathbf{2} \text { power } \\
\text { levels }\end{array}$ & $\begin{array}{c}\mathbf{4} \text { power } \\
\text { levels }\end{array}$ & $\begin{array}{c}\mathbf{5} \text { power } \\
\text { levels }\end{array}$ \\
\hline avg-user-Th (kbps) & 316.2 & 330 & 331.1 \\
Gain \% & $\mathbf{+ 4 . 8 \%}$ & $\mathbf{+ 9 . 4 \%}$ & $\mathbf{+ 9 . 8 \%}$ \\
\hline cell-edge-Th (kbps) & 43.7 & 55 & 61.3 \\
Gain \% & $\mathbf{+ 1 2 . 4 \%}$ & $\mathbf{+ 4 1 . 5 \%}$ & $\mathbf{+ 5 7 . 9 \%}$ \\
\hline avg-EE (bits/joule) & 228 & 217.2 & 218.1 \\
Gain \% & $\mathbf{+ 1 9 . 6 \%}$ & $\mathbf{+ 1 3 . 9 \%}$ & $\mathbf{+ 1 4 . 4 \%}$ \\
\hline cell-edge-EE (bits/joule) & 25.7 & $\mathbf{2 4 . 3}$ & 25.2 \\
Gain \% & $\mathbf{+ 2 5 \%}$ & $\mathbf{+ 1 8 . 4 \%}$ & $\mathbf{+ 2 3 \%}$ \\
\hline \multicolumn{2}{|c}{}
\end{tabular}

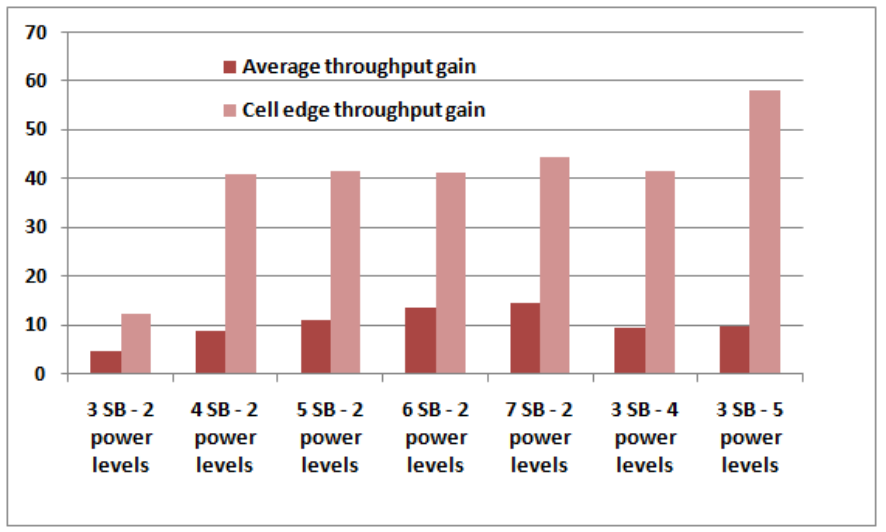

Fig. 5. Average and cell edge throughput gains - macros only scenario.

only 3 sub-bands. The gain from the 3 sub-bands to the next configurations is more important for cell edge users.

\section{Disjoint Power Levels Optimization}

We fix the number of sub-bands to 3 and study the impact of power optimization. We have the following three cases:

- 2 power levels per RB: 0 and 0.8 watts for MC and 0 and 0.02 watts for SC.

- 4 power levels per RB: 0, 0.4, 0.8 and 1.2 watts for MC and $0,0.01,0.02$ and 0.03 watts for SC.

- 5 power levels per RB: 0, 0.4, 0.8, 1.2 and 1.6 watts for $\mathrm{MC}$ and $0,0.01,0.02,0.03$ and 0.04 watts for SC.

The results are given in Table IV and V. The gains are computed in comparison to the frequency static reuse-1 configuration.

The simulation results show that:

- Power level optimization further improves the cell edge user throughput. We notice that by increasing from 2 power levels to 5 power levels, we get $45 \%$ more gain in macros only scenario and 30\% more gain in HetNets.

- The average user throughput is quite the same in the three different configurations for HetNets and slightly increases for macros only.

- The average and cell edge energy efficiencies are more or less decreasing when using more power levels. It means that the network is transmitting with more power than 
TABLE V

POWER LEVELS OPTIMIZATION RESUlt - HETNETS

\begin{tabular}{|c||c|c|c|}
\hline $\boldsymbol{F = 3 , ~} \boldsymbol{T = 1}$ & $\begin{array}{c}\mathbf{2} \text { power } \\
\text { levels }\end{array}$ & $\begin{array}{c}\mathbf{4} \text { power } \\
\text { levels }\end{array}$ & $\begin{array}{c}\mathbf{5} \text { power } \\
\text { levels }\end{array}$ \\
\hline avg-user-Th (kbps) & 458.4 & 458.6 & 465 \\
Gain \% & $\mathbf{+ 2 0 . 1 \%}$ & $\mathbf{+ 2 0 . 2 \%}$ & $\mathbf{+ 2 1 . 9 \%}$ \\
\hline cell-edge-Th (kbps) & 49.5 & 56.9 & 62 \\
Gain \% & $\mathbf{+ 2 2 . 6 \%}$ & $\mathbf{+ 4 0 . 9 \%}$ & $\mathbf{+ 5 3 . 6 \%}$ \\
\hline avg-EE (bits/joule) & 583.1 & 485.4 & 470.6 \\
Gain \% & $\mathbf{+ 5 9 . 5 \%}$ & $\mathbf{+ 3 2 . 8 \%}$ & $\mathbf{+ 2 8 . 7 \%}$ \\
\hline cell-edge-EE (bits/joule) & 31.1 & 28.3 & 27.3 \\
Gain \% & $\mathbf{+ 5 1 . 2 \%}$ & $\mathbf{+ 3 7 . 7 \%}$ & $\mathbf{+ 3 3 \%}$ \\
\hline
\end{tabular}

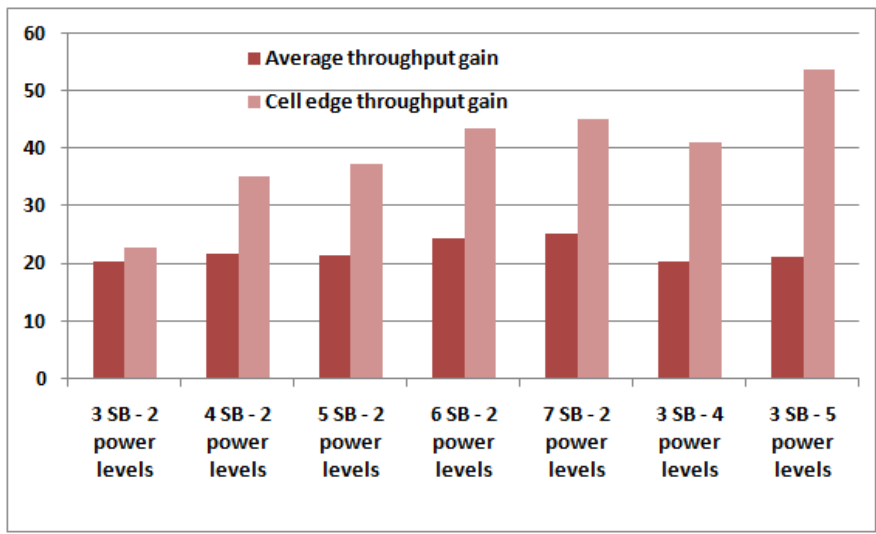

Fig. 6. Average and cell edge throughput gains - HetNets scenario.

the case of only 2 power levels. Generally speaking, the optimal settings are either to boost the power on some sub-bands or to choose a low power level different from zero. As the average throughput is almost stable and the average energy efficiency is decreasing, we can say that the network is consuming more power but not transmitting more data in the system. However, the cell edge user throughput is increasing when using more power levels.

Fig. 5 and Fig. 6 overview the average and cell edge throughput gains in the macros only and HetNets scenarios, respectively. We regroup all the frequency sub-band and power levels optimization results for comparison. Generally speaking, further enhancement can be expected at the cost of higher computation complexity.

\section{Time Domain Optimization via ABS}

The time resource optimization via Almost Blank Subframes consists in defining for each macro cell a power pattern of 40 sub-frames over the whole bandwidth. In the muted subframes, i.e., when corresponding power pattern equals to 0 , the macro eNB does not send any traffic channels. To limit the number of possible configurations, we regroup the sub-frames into equal sized slots.

We simulate 2 configurations of HetNets scenario:

- 4 time slots, each containing 10 sub-frames,
TABLE VI

DISJOINT ABS OPTIMIZATION RESULT

\begin{tabular}{|c||c|c|c|}
\hline $\boldsymbol{F = 1}$ & $\begin{array}{c}\text { No } \\
\text { optim }\end{array}$ & $\boldsymbol{T = 4}$ & $\boldsymbol{T = 8}$ \\
\hline avg-user-Th (kbps) & 381.4 & 442.3 & 461.3 \\
Gain \% & - & $\mathbf{+ 1 5 . 9 \%}$ & $\mathbf{+ 2 0 . 9 \%}$ \\
\hline cell-edge-Th (kbps) & 40.4 & 49.7 & 54.9 \\
Gain \% & - & $\mathbf{+ 2 2 . 9 \%}$ & $\mathbf{+ 3 5 . 9 \%}$ \\
\hline avg-EE (bits/joule) & 365.6 & 527.8 & 541.3 \\
Gain \% & - & $\mathbf{+ 4 4 . 3 \%}$ & $\mathbf{+ 4 8 \%}$ \\
\hline cell-edge-EE (bits/joule) & 20.5 & 29.6 & 31.5 \\
Gain \% & - & $\mathbf{+ 4 3 . 9 \%}$ & $\mathbf{+ 5 3 . 6 \%}$ \\
\hline
\end{tabular}

- 8 time slots and each slot is composed of 5 sub-frames. The simulations results are summarized in Table VI along with the case where no ABS optimization is applied.

The disjoint ABS optimization in HetNets scenario enhances the average and cell edge performance, as we obtain:

- a gain of $15 \%$ and $22 \%$ in average and cell edge user throughput respectively, using the 4 time slots optimization. This imrovement is even more important with the second simulation configuration, i.e., using 8 time slots of 5 sub-frames. Choosing the optimal ABS for each macro cell, permits to mitigate the inter cell interference and enhance the average and cell edge users rates. Muting certain macro cells during some time slots, allows their neighboring SC to schedule their attached UEs, which are strongly interfered by the $\mathrm{MC}$, in the protected sub-frames. The other UEs located at the cell center are scheduled over all sub-frames since the interference experienced from the macro eNBs is negligible compared to the signal of the SC.

- about $50 \%$ of gain in average and cell edge energy efficiency. The increase of the bits per joule is much important than the throughput gains. This implies that with the ABS optimization, the cells are transmitting more data using lower power during the simulation. Muting some macro cells permits to decrease the power consumption but not at the cost of users throughput.

\section{E. User Association via CIO \& Resource Allocation Optimiza- tion}

To study the impact of user association using cell individual offset, we made multiple simulations using the first scenario, i.e., all the users are initially attached to macro cells. The results are presented in Table VII for various configurations:

- Static reuse of 1 and no optimization is performed in the coordinator,

- Disjoint user association optimization using CIOs,

- Joint CIO optimization and Frequency sub-band optimization.

When performing disjoint CIO optimization, the average user throughput of the network increases slightly: from 301 to $321 \mathrm{kbps}$. This is due to the change in CIO values and hence 
TABLE VII

User Association and Resource Allocation Optimization RESULT

\begin{tabular}{|c|c|c|c|c|}
\hline$T=1$ & $\begin{array}{c}\text { Static } \\
\text { reuse-1 }\end{array}$ & $\begin{array}{c}\text { CIO optim } \\
F=1\end{array}$ & $\begin{array}{c}\text { CIO optim } \\
F=3\end{array}$ & $\begin{array}{c}\text { CIO optim } \\
F=4\end{array}$ \\
\hline $\begin{array}{l}\text { Avg-user-Th } \\
\text { (kbps) }\end{array}$ & 301.7 & 321.7 & 349.6 & 360 \\
\hline Gain \% & - & $+6.7 \%$ & $+15.9 \%$ & $+19.4 \%$ \\
\hline $\begin{array}{l}\text { Cell-edge-Th } \\
\quad(\mathrm{kbps})\end{array}$ & 38.9 & 34.7 & 40.1 & 49.5 \\
\hline Gain \% & - & $-10.7 \%$ & $+3.3 \%$ & $+27.4 \%$ \\
\hline $\begin{array}{c}\text { Avg-EE } \\
\text { (bits/joule) }\end{array}$ & 190.7 & 200.7 & 255 & 282.1 \\
\hline Gain \% & - & $+5.2 \%$ & $+\mathbf{3 3 . 8 \%}$ & $+48 \%$ \\
\hline $\begin{array}{c}\text { Cell-edge-EE } \\
\text { (bits/joule) }\end{array}$ & 20.5 & 18.9 & 23.4 & 27.5 \\
\hline Gain \% & - & $-8.1 \%$ & $+56.7 \%$ & $+54.7 \%$ \\
\hline
\end{tabular}

associating some UEs to SCs offering them higher bandwidth. However, the cell edge users remain highly interfered by MCs which continue to transmit with maximum power over the bandwidth as there is no interference mitigation in this case. This strong inter-cell interference is the reason behind the decrease of the cell edge throughput compared to the first configuration where there is no CIO optimization.

The best performances are given by the joint optimization of $\mathrm{CIO}$ and frequency sub-band optimization. We can see that the gain obtained for the average throughput reaches $20 \%$ and for cell edge throughput is around $27 \%$. This optimization allows to offload traffic from MCs and to have an efficient distribution of the resources among the neighboring cells which leads to better users experience.

\section{CONClusion AND Future Work}

Based on potential game setup, we offer a practical solution to optimize user association and coordinate inter-cell interference among multiple cells in LTE. The algorithm can provide optimal cell individual offsets and power settings over frequency and time resources for each cell to maximize a network utility. We observe that the proposed algorithm outperforms the frequency reuse- 1 scheme and achieves more than 50\% gain in cell edge throughput and also substantial enhancement in the average throughput and energy efficiency. From simulation, we see that the algorithm converges to a Nash equilibrium point and only requires a small number of iterations. This would allow us to optimize a dynamic system due to its fast convergence. Since each iteration follows the best response greedy strategy, the network is always improving by the proposed schemes towards the optimal solution.

The presented method can be also useful for other resource allocation optimization problems and different criteria of similar systems. As future work, we plan to:

- extend our solution under mobility by adding a cost associated to LTE/LTE-A Mobility Robustness Optimization (MRO) indicators in the network utility formulation.
Another interesting direction is to allow each eNB to use different CIOs with respect to various classes of UE velocities. This new configuration could provide a dynamic solution of user association by selecting the best CIOs based on mobility class.

- integrate the traffic profile of the users and consider user satisfaction or the quality of experience (QoE). Some QoE parameters can be integrated to the virtual scheduler for example to take into account certain required minimum throughput for each user.

- regroup the eNBs in clusters and distribute the computation tasks correspondingly. It is possible to have fully centralized, distributed or hybrid architectures, using the same optimization approach as in the paper. Obviously, there are trade-offs among the computational complexity in each cluster depending on its size, the amount of message exchanges, and the performance loss from a centralized to a fully distributed architecture.

\section{ACKNOWLEDGMENT}

This work is under the common lab between INRIA and Bell Labs. A part of the work was carried out at LINCS (www.lincs.fr). It was supported by ANR project IDEFIX under grant ANR-13-INFR-0006 and the European Union's Seventh Framework Programme (FP7/2007-2013) under grant no. 318115 (Connectivity management for eneRgy Optimized Wireless Dense networks CROWD). A part of the work [3] was presented at IEEE/IFIP Network Operations and Management Symposium (NOMS) 2016.

\section{REFERENCES}

[1] ETSI TS 36.300 v10.11.0 LTE; Evolved Universal Terrestrial Radio Access (E-UTRA) and Evolved Universal Terrestrial Radio Access Network (E-UTRAN); Overall description; Stage 2 (3GPP TS 36.300 version 10.11.0 Release 10)

[2] S. Sesia, I. Toufik, and M. Baker, LTE - The UMTS Long Term Evolution: From Theory to Practice, 2nd Edition, Wiley, 2011.

[3] N. Trabelsi, C. S. Chen, L. Roullet, E. Altman and R. El Azouzi, "Scheduling via Frequency and Power Allocation Optimization in LTE Cellular Networks," IEEE/IFIP Network Operations and Management Symposium (NOMS), April, 2016.

[4] C. S. Chen, F. Baccelli, and L. Roullet, "Joint Optimization of Radio Resources in Small and Macro Cell Networks," IEEE Vehicular Technology Conference (VTC), May 2011.

[5] A S. Hamza, S S. Khalifa, H S. Hamza, and K. El-Sayed, "A Survey on Inter-Cell Interference Coordination Techniques in OFDMA-Based Cellular Networks," IEEE Communications Surveys and Tutorials, vol. 15, no. 4, pp. 1642-1670, March 2013.

[6] A. Mills, D. Lister, and M. De Vos, "Understanding Static Inter-Cell Interference Coordination Mechanisms in LTE," Journal of Communications, vol. 6, no. 4, July 2011.

[7] E. Pateromichelakis, M. Shariat, A. ul Quddus, and R. Tafazolli, "On the Evolution of Multi-Cell Scheduling in 3GPP LTE/LTE-A," IEEE Communications Surveys and Tutorials, 2012.

[8] R. Agrawal, A S. Bedekar, S. Kalyanasundaram, N. Arulselvan, T E. Kolding, and H. Kroener, "Centralized and Decentralized Coordinated Scheduling with Muting," IEEE Vehicular Technology Conference (VTC), May 2014.

[9] Md. Shipon Ali, "An overview on Interference Management in 3GPP LTE-advanced Heterogeneous Networks," International Journal of Future Communication and Networking, vol. 8, no. 1, 2015. 
[10] Q. Ye, M. Al-Shalash, C. Caramanis, and J. G. Andrews, "On/off macrocells and load balancing in heterogeneous cellular networks," IEEE Global Communications Conference (GLOBECOM), pp. 38143819, 2013.

[11] Y. Jin and L. Qiu, "Joint user association and interference coordination in heterogeneous cellular networks," IEEE Commun. Lett., vol. 17, no. 12, pp. 22962299, 2013.

[12] D. Lopez-Perez, I. Guvenc, G. de la Roche, M. Kountouris, T. Q. S. Quek and J. Zhang, "Enhanced intercell interference coordination challenges in heterogeneous networks," IEEE Wireless Communication Magazine, vol. 18, no. 3, pp. 22-30, June 2011.

[13] Kyocera, "Potential performance of range expansion in macro-pico deployment (r1-104355)," 3GPP TSG RAN WG1 Meeting-62, August 2010.

[14] N. Trabelsi, L. Roullet, and A. Feki, "A Generic Framework for Dynamic eICIC Optimization in LTE Heterogeneous Networks," IEEE Vehicular Technology Conference (VTC), September 2014

[15] L. Lindbom, R. Love, S. Krishnamurthy, C. Yao, N. Miki and V. Chandrasekhar, "Enhanced inter-cell interference coordination for heterogeneous networks in LTE-advanced:A survey,"Cornell University Library, December 2011.

[16] ETSI TS 36.300, "LTE Evolved Universal Terrestrial Radio Access (EUTRA) and Evolved Universal Terrestrial Radio Access Network (EUTRAN); Overall Description; Stage 2,” Tech Spec. v10.11.0, Sep. 2013.

[17] D. Liu, L. Wang, Y. Chen, M. Elkashlan, K.-K. Wong, R. Schober and L. Hanzo, "User association in 5G networks: A survey and an outlook," IEEE Communications Surveys \& Tutorials, January 2016.

[18] Q. Ye, B. Rong, Y. Chen, M. Shalash, C. Caramanis, and J. G. Andrews, "User association for load balancing in heterogeneous cellular networks, IEEE Trans. on Wireless Communications, vol. 12, no. 6, pp. 27062716, June 2013.

[19] D. Monderer and L. S. Shapley, "Potential Games," Games and Economic Behavior, vol. 14, no. 1, pp. 124-143, May 1996.

[20] A. Gopalasingham, L. Roullet, N. Trabelsi, C.S. Shen, A. Hebbar, E. Bizouarn, "Generalized Software Defined Network Platform forRadio Access Networks," IEEE Consumer Communications \& Networking Conference (CCNC), January 2016.

[21] C. Mehlfuhrer, J. C. Ikuno, M. Simko, S. Schwarz, M. Wrulich, and M. Rupp, "The Vienna LTE Simulators - Enabling Reproducibility in Wireless Communications Research," EURASIP Journal on Advances in Signal Processing, July 2011.

Nessrine Trabelsi received a double Engineering degree from Telecom SudParis (France) and from The National School of Computer Science (University of Manouba, Tunisia) in 2011. In 2012, She received the M.Sc degree in computer science from The National School of Computer Science. From 2013 to 2016, she was part of the common lab of The National Institute for Research in Computer Science and Control (Inria) and Bell Labs Nokia France, to prepare her Ph.D degree that she obtained in 2016

from the University of Avignon. Her research focuses on on small cells optimization, user association and interference coordination in LTE cellular networks.

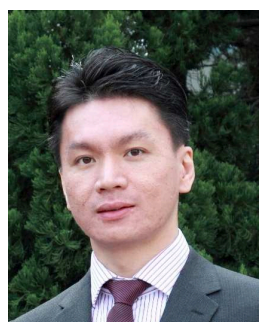

Chung Shue Chen (S'02-M'05-SM'16) received the B.Eng., M.Phil., and Ph.D. degrees in information engineering from The Chinese University of Hong Kong in 1999, 2001, and 2005, respectively.

$\mathrm{He}$ is a Member of Technical Staff (MTS) at Nokia Bell Labs. Prior to joining Bell Labs, he worked at INRIA, in the research group on Network Theory and Communications (TREC, INRIA-ENS). He was an Assistant Professor at The Chinese University of Hong Kong (CUHK). He was an ERCIM Fellow at Norwegian University of Science and Technology (NTNU), Norway, and National Center for Mathematics and Computer Science (CWI), The Netherlands.

His research interests include wireless communications and networking, radio resource management, self-organizing networks, and optimization algorithms. He has served as TPC in international conferences including IEEE ICC, Globecom, WCNC, VTC, CCNC, and WiOpt. He is an Editor of Transactions on Emerging Telecommunications Technologies (ETT) since 2011. He also holds a position of permanent member at Laboratory of Information, Networking and Communication Sciences (LINCS), in Paris. He was the recipient of Sir Edward Youde Memorial Fellowship and ERCIM Alain Bensoussan Fellowship.

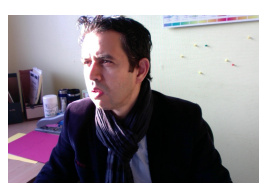

Rachid El-Azouzi received the Ph.D. degree in Applied Mathematics from the Mohammed V University, Morocco (2000). He joined INRIA Sophia Antipolis for post-doctoral and Research Engineer positions. Since 2003, he has been a researcher at the University of Avignon, France. Currently he serves on the editorial board of IEEE Transaction on Networking. His research interests are research interests are, Networking Games, Networking games, Design of communication protocols for wireless network, Intelligent wireless networks and learning algorithms, MANET networks and Delay Tolerant Networking and Mean Field.

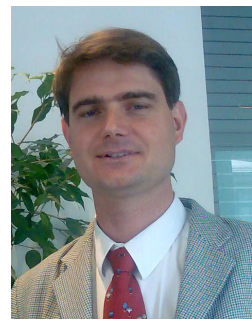

Laurent Roullet (male) is Head of Virtualization and Verification Group in Nokia Bell-Labs. He is leading Activities focusing on Cloud RAN architectures, front-hauling optimization, Software Defined Networks and Network Function Virtualization applied to wireless access, both $4 \mathrm{G}$ and $5 \mathrm{G}$. $\mathrm{He}$ joined Bell Labs in 2010 and has worked on small cells optimization and interference coordination. $\mathrm{He}$ joined Alcatel Lucent Mobile Broadcast in 2006 and led the standardization effort of the DVB-SH (hybrid satellite terrestrial mobile broadcast). From 2001 to 2005 he worked in UDcast, a start-up focusing on mobile routers for satellite and DVB networks and developed the first DVB-H infrastructure in cooperation with Nokia. From 1997 to 2001 he worked in Alcatel Space on onboard processing. Laurent Roullet is graduated from Ecole Polytechnique and SupAero. 
Eitan Altman received the B.Sc. degree in electrical engineering (1984), the B.A. degree in physics (1984) and the $\mathrm{Ph} . \mathrm{D}$. degree in electrical engineering (1990), all from the Technion-Israel Institute, Haifa. In 1990 he received his B.Mus. degree in music composition in Tel-Aviv university. Since 1990, Dr. Altman has been a researcher at INRIA (National research institute in computer science and control). He has been in the editorial boards of the journals Wireless Networks (WINET), Computer Networks (COMNET), Computer Communications (Comcom), J. Discrete Event Dynamic Systems (JDEDS), SIAM J. of Control and Optimisation (SICON), Stochastic Models, and Journal of Economy Dynamic and Control (JEDC). He received the best paper award in the Networking 2006, in Globecom 2007, in IFIP Wireless Days 2009 and in CNSM 2011 (Paris) conferences. His areas of interest include Network Engineering Games, social networks and their control and the analysis through game theoretical models of network neutrality issues. He received in 2012 the Grand Prix de France Telecom from the French Academy of Sciences. On July 2014 he received the ISAACS Award from the Society of Dynamic Games for his contribution in game Theory. More information can be found at www-sop.inria.fr/members/Eitan.Altman/ 